NOTICE: This is the author's version of a work that was accepted for publication in Advances in Space Research. Changes resulting from the publishing process, such as peer review, editing, corrections, structural formatting, and other quality control mechanisms may not be reflected in this document. Changes may have been made to this work since it was submitted for publication. A definitive version was subsequently published in Advances in Space Research [46, 2, 2010] DOI 10.1016/j.asr.2010.02.023 


\title{
Testing a New Multivariate GNSS Carrier Phase Attitude Determination Method for Remote Sensing Platforms
}

\author{
Gabriele Giorgi* \\ Delft Institute of Earth Observation and Space Systems (DEOS), \\ Delft University of Technology, PO Box 5058, 2600 GB Delft, The Netherlands \\ Peter J. G. Teunissen \\ Department of Spatial Sciences, Curtin University of Technology, \\ GPO Box U1987, Perth WA 6845, Australia \\ Delft Institute of Earth Observation and Space Systems (DEOS), \\ Delft University of Technology, PO Box 5058, 2600 GB Delft, The Netherlands \\ Sandra Verhagen, Peter J. Buist \\ Delft Institute of Earth Observation and Space Systems (DEOS), \\ Delft University of Technology, PO Box 5058, 2600 GB Delft, The Netherlands
}

\begin{abstract}
GNSS-based Attitude Determination is an important field of study, since it is a valuable technique for the orientation estimation of remote sensing platforms. To achieve highly accurate angular estimates, the precise GNSS carrier phase observables must be employed. However, in order to take full advantage of the high precision, the unknown integer ambiguities of the carrier phase observables need to be resolved. This contribution presents a GNSS carrier phase-based attitude determination method that determines the integer ambiguities and attitude in an integral manner, thereby fully exploiting the known body geometry of the multi-antennae configuration. It is shown that this integral approach aids the ambiguity resolution process tremendously and strongly improves the capacity of fixing the correct set of integer ambiguities. In this contribution the challenging scenario of single-epoch, single-frequency attitude determination is addressed. This guarantees a total independence from carrier phase slips and losses of lock, and it also does not require any a-priori motion model for the platform. The method presented is a multivariate constrained version of the popular LAMBDA method and it is tested on data collected during an airborne remote sensing campaign.
\end{abstract}

Key words: GNSS, Attitude determination, Multivariate Constrained LAMBDA 


\section{Introduction}

GNSS (Global Navigation Satellite Systems) technology is a valid aid in support of Earth Observation sciences, both to provide platform navigation and as an additional sensing instrument (Beutler et al., 1999). GNSS positioning and navigation have been successfully employed in a number of airborne imagery and mobile mapping campaigns (Corbett, 1993; Kocaman, 2003; Legat et al., 2006), as well as in a number of recent spaceborne earth observation missions (Bock et al., 2002, 2007; Kang et al., 2003; Montenbruck et al., 2008), providing an accurate estimate of the platform's absolute position and attitude. GNSS signals have been exploited to study various atmosphere parameters, through the analysis of their reflections or deflections in the different layers of the earth atmosphere (Azpilicueta et al., 2006; Jin et al., 2007; Jin and Luo, 2009; Knedlik et al., 2008; Ruzhin et al., 1998). Also remote sensing campaigns conducted by means of unmanned airborne vehicles (UAVs) or formation flying satellites widely benefit from the GNSS technology.

One of the main issues in remote sensing applications is the precise orientation estimation of the platform which carries the sensors (such as radars and lasers). Many sensors and technologies are available to estimate the attitude of a platform, but there is a growing interest in GNSS-based Attitude Determination (AD), often integrated at various levels of tightness to other types of sensors, typically Inertial Measurements Units (IMU). Although the accuracy of a stand-alone GNSS attitude system might not be comparable with the one obtainable with other modern attitude sensors, a GNSS-based system presents several advantages: it is inherently driftless, minor maintenance is required and it is not as expensive as other high-precision systems, such as INS and Star Trackers. Several studies have been carried out to investigate the feasibility and performance of GNSS-based Attitude Determination, see e.g. (Axelrad and Ward, 1994; Bar-Itzhack et al., 1998; Brown, 1992; Cohen, 1992; Crassidis et al., 1997; Dai et al., 2004; Euler, 1995; Giorgi and Buist, 2008; Hauschild and Montenbruck, 2007; Kim and Langley, 2000; Kuylen et al., 2005; Li et al., 2004; Madsen and Lightsey, 2004; Monikes et al., 2005; Psiaki, 2006; Schleppe, 1997).

The precision of GNSS-based attitude determination is driven by the quality of the GNSS observations and the length of the baselines between the antennae. A precise angular estimate is obtained exploiting the GNSS carrier phase observables, which are two orders of magnitude more accurate than the GNSS code observables. The carrier phase measurements are, however, affected by unknown integer ambiguities, since only their fractional part is measured by the receiver. Due to its computational efficiency, the LAMBDA (Least-squares

\footnotetext{
* Corresponding author

Email address: G.Giorgi@TUDelft.nl (Gabriele Giorgi).
} 
AMBiguity Decorrelation Adjustment) method (Teunissen, 1994a) is currently a widely used method for Ambiguity Resolution (AR). The method is an implementation of the optimal Integer Least-Squares (ILS) (Teunissen, 1994b, 1999) principle.

Although the standard LAMBDA method has been applied to AD applications, see e.g. (Kuylen et al., 2006; Monikes et al., 2005; Wang et al., 2009), the intrinsic properties of the $\mathrm{AD}$ problem have not been fully integrated in these works. In (Kuylen et al., 2006), for instance, the known baseline length was only used as validation step and in (Monikes et al., 2005; Wang et al., 2009), the (single) baseline length constraint was used to modify the LAMBDA search routines for a subset of the unknown integer ambiguities. In all existing approaches, however, the complete set of a-priori information is not integrally exploited to directly aid the ambiguity resolution process. In this contribution a novel algorithm based on a nontrivial modification of the LAMBDA method is presented and tested. The method solves for the GNSS integer ambiguities and the attitude of the platform in an integral manner, thereby fully exploiting the set of nonlinear geometry constraints available. This Multivariate Constrained LAMBDA method (MC-LAMBDA), theoretically introduced in (Teunissen, 2007a), has numerous advantages: it is applicable to any number of antennae, to any GNSS system and combinations of them, to any number of frequencies, and it does not need any a-priori information about the attitude or the dynamics of the platform. The MC-LAMBDA method is reviewed and its performance is tested by processing and analysing data collected during an airborne gravimetry experiment.

This contribution is structured as follows. In Section 2, the GNSS Attitude model is presented, while its multivariate constrained integer least-squares solution is given in Section 3. The results obtained from testing the method, on both static and dynamic platforms, are presented and discussed in Section 4. It is emphasized that we address in this contribution the most challenging AR scenario, namely single-epoch, single-frequency AR. This guarantees a total independence from carrier phase slips and losses of lock, and it also does not require any a-priori motion model for the platform.

\section{The GNSS-based Attitude model}

The phase and code GNSS observations collected at time $t$ at receiver $r$ tracking satellite $s$ are modeled as (Teunissen and Kleusberg, 1998)

$$
\begin{aligned}
P_{r}^{s}(t)= & \rho_{r}^{s}\left(t, t-\tau_{r}^{s}\right)+I_{r}^{s}+T_{r}^{s}+d m_{r}^{s}+c\left[d t_{r}(t)-d t^{s}\left(t-\tau_{r}^{s}\right)\right]+ \\
& c\left[d_{r}(t)+d^{s}\left(t-\tau_{r}^{s}\right)\right]+e_{r}^{s}
\end{aligned}
$$




$$
\begin{aligned}
\Phi_{r}^{s}(t)= & \rho_{r}^{s}\left(t, t-\tau_{r}^{s}\right)-I_{r}^{s}+T_{r}^{s}+\delta m_{r}^{s}(t)+c\left[d t_{r}(t)-d t^{s}\left(t-\tau_{r}^{s}\right)\right]+ \\
& c\left[\delta_{r}(t)+\delta^{s}\left(t-\tau_{r}^{s}\right)\right]+\lambda\left[\phi_{r}\left(t_{0}\right)+\phi^{s}\left(t_{0}\right)\right]+\lambda N_{r}^{s}+\varepsilon_{r}^{s}
\end{aligned}
$$

where

$$
\begin{array}{ll}
P(t), \Phi(t) & : \text { code and phase observations at time } t[\mathrm{~m}] \\
\tau & : \text { signal travel time satellite-receiver }[\mathrm{s}] \\
\rho & : \text { geometrical distance between receiver and satellite }[\mathrm{m}] \\
I, T & : \text { ionospheric and tropospheric effects }[\mathrm{m}] \\
d m, \delta m & : \text { code and phase multipath errors }[\mathrm{m}] \\
c & : \text { speed of light }\left(299792458 \frac{\mathrm{m}}{\mathrm{s}}\right) \\
d t & : \text { clock errors }[\mathrm{s}] \\
d, \delta & : \text { instrumental delays }[\mathrm{s}] \\
\phi & : \text { phase of the generated carrier signal (original or replica) }[\mathrm{rad}] \\
\lambda & : \text { carrier phase wavelength }[\mathrm{m}] \\
e, \varepsilon & : \text { remaining unmodeled errors }[\mathrm{m}]
\end{array}
$$

For those applications where one is interested in estimation of the relative positions of antennae rather than in their absolute positions, the differences between observations taken at the same time, from the same satellite $s$, at different receivers $r_{1}$ and $r_{2}$ (i.e. Single-Differences, SD) are formed as

$$
\begin{aligned}
P_{r_{12}}^{s}(t)= & \rho_{r_{2}}^{s}\left(t, t-\tau_{r_{2}}^{s}\right)-\rho_{r_{1}}^{s}\left(t, t-\tau_{r_{1}}^{s}\right)+I_{r_{12}}^{s}+T_{r_{12}}^{s}+d m_{r_{12}}^{s}+c d t_{r_{12}}+ \\
& +c d_{r_{12}}+e_{r_{12}}^{s} \\
& \\
\Phi_{r_{12}}^{s}(t)=\quad & \rho_{r_{2}}^{s}\left(t, t-\tau_{r_{2}}^{s}\right)-\rho_{r_{1}}^{s}\left(t, t-\tau_{r_{1}}^{s}\right)-I_{r_{12}}^{s}+T_{r_{12}}^{s}+\delta m_{r_{12}}^{s}+c d t_{r_{12}}+ \\
& +c \delta_{r_{12}}+\lambda \phi_{r_{12}}\left(t_{0}\right)+\lambda N_{r_{12}}^{s}+\varepsilon_{r_{12}}^{s}
\end{aligned}
$$

where $(\cdot)_{r_{12}}=(\cdot)_{r_{2}}-(\cdot)_{r_{1}}$. Via the differencing operation, many terms cancel out, like the (common) phase term relative to the common satellite $s$ and the instrumental delays and clock errors of satellite $s$.

The dimensionless term $N_{r_{12}}^{s}$ indicates a whole number of cycles: it quantifies the integer part of the measured phase difference between two receivers, the so-called integer ambiguity.

When addressing the AD problem, the SDs are taken between antennae placed onboard a platform, and typically the size of the body (ship, land vehicle, aircraft or space platform) is less than a few hundreds of meters. This allows one to neglect the atmospheric effects, which have very small variations on such short baselines. The clock biases and the different instrumental delays still have to be accounted for.

To eliminate the remaining clock terms, the Double-Differences (DD), i.e. the differences between observations taken at the same time, from two satellites, 
at different receivers, are formed as

$$
\begin{aligned}
P_{r_{12}}^{s_{12}}(t)= & \rho_{r_{2}}^{s_{2}}\left(t, t-\tau_{r_{2}}^{s_{2}}\right)-\rho_{r_{1}}^{s_{2}}\left(t, t-\tau_{r_{1}}^{s_{2}}\right)-\rho_{r_{2}}^{s_{1}}\left(t, t-\tau_{r_{2}}^{s_{1}}\right)+\rho_{r_{1}}^{s_{1}}\left(t, t-\tau_{r_{1}}^{s_{1}}\right)+ \\
& +d m_{r_{12}}^{s_{12}}+e_{r_{12}}^{s_{12}} \\
\Phi_{r_{12}}^{s_{12}}(t)= & \rho_{r_{2}}^{s_{2}}\left(t, t-\tau_{r_{2}}^{s_{2}}\right)-\rho_{r_{1}}^{s_{2}}\left(t, t-\tau_{r_{1}}^{s_{2}}\right)-\rho_{r_{2}}^{s_{1}}\left(t, t-\tau_{r_{2}}^{s_{1}}\right)+\rho_{r_{1}}^{s_{1}}\left(t, t-\tau_{r_{1}}^{s_{1}}\right)+ \\
& +\delta m_{r_{12}}^{s_{12}}+\lambda N_{r_{12}}^{s_{12}}+\varepsilon_{r_{12}}^{s_{12}}
\end{aligned}
$$

where $(\cdot)_{r_{12}}^{s_{12}}=(\cdot)_{r_{12}}^{s_{2}}-(\cdot)_{r_{12}}^{s_{1}}$. The advantage of the double differences lies in the reduced set of unknowns, namely only the baseline coordinates and the integer ambiguities remain. In this contribution multipath is not corrected or modeled for, so it is lumped in the terms $e$ and $\varepsilon$. The geometrical term $\rho$ in (5) and (6) contains the information about the satellites-receivers geometry, but a linearization step is necessary to extract the three sought for baseline coordinates. Using $\rho_{r}^{s}=\left\|r^{s}-r_{r}\right\|$, where $r^{s}$ is the satellite position vector and $r_{r}$ the receiver position vector, the linearized expressions for (5) and (6) read (Teunissen and Kleusberg, 1998)

$$
\begin{gathered}
\Delta P_{r_{12}}^{s_{12}}=\left(-u_{r_{12}}^{s_{12}}\right)^{T} \Delta r_{12} \\
\Delta \Phi_{r_{12}}^{s_{12}}=\left(-u_{r_{12}}^{s_{12}}\right)^{T} \Delta r_{12}+\lambda N_{r_{12}}^{s_{12}}
\end{gathered}
$$

where we dropped the time dependence notation. $\Delta P, \Delta \Phi$ stand for the 'observed minus computed' observations, $\Delta r_{12}$ is the increment vector of the baseline coordinates and $u_{r_{12}}^{s_{12}}$ is the DD unit line-of-sight vector. In order to simplify the notation, the set of $2 n$ observations collected tracking $n+1$ satellites on a single frequency is grouped into the $(2 n)$-vector of observed minus computed code and carrier phase measurements:

$$
y=\left[\Delta P_{r_{12}}^{s_{1 k}}, \cdots, \Delta P_{r_{12}}^{s_{n k}}, \Delta \Phi_{r_{12}}^{s_{1 k}}, \cdots, \Delta \Phi_{r_{12}}^{s_{n k}}\right]^{T}
$$

where the DDs are formed taking satellite $k$ as reference. The linearized set of DD GNSS code and phase observations tracking $n+1$ satellites on a single frequency is then cast into the model

$$
\begin{aligned}
& E(y)=A z+G b \quad z \in \mathbb{Z}^{n} ; b \in \mathbb{R}^{3} \\
& D(y)=Q_{y}
\end{aligned}
$$

where $E(\cdot)$ is the expectation operator, $z$ contains the $n$ unknown integervalued ambiguities $\left[N_{r_{12}}^{s_{1 k}} \cdots N_{r_{12}}^{s_{n k}}\right]^{T}$ and $b$ is the vector of real-valued baseline coordinates. $A$ is the $2 n \times n$ matrix which contains the carrier wavelength, 
while $G$ is the $2 n \times 3$ matrix of normalized DD line-of-sight vectors:

$$
A=\left[\begin{array}{ll}
\mathbf{0} & \\
& \lambda I_{n}
\end{array}\right] \quad G=\left[\begin{array}{c}
\left(-u_{r_{12}}^{s_{1 k}}\right)^{T} \\
\vdots \\
\left(-u_{r_{12}}^{s_{n k}}\right)^{T}
\end{array}\right]
$$

$D(\cdot)$ is the dispersion operator: a Gaussian-distributed error is assumed on the vectors of observables, characterized by the variance-covariance (v-c) matrix $Q_{y}$. The integer nature of the $n$ ambiguities is made clear through the notation $z \in \mathbb{Z}^{n}$, while the baseline vector $b$ belongs to the space of real vectors $b \in \mathbb{R}^{3}$. In (Teunissen, 2007a) it was shown how to extend model (10) if a set of $m+1$ antennae collects observations all tracking the same $n+1$ satellites:

$$
\begin{aligned}
E(Y) & =A Z+G B \quad Z \in \mathbb{Z}^{n \times m} ; B \in \mathbb{R}^{3 \times m} \\
D(\operatorname{vec}(Y)) & =Q_{Y}
\end{aligned}
$$

where $Y$ is the $2 n \times m$ matrix whose columns are the linearized DD code and phase observations of each baseline, $Z$ is the $n \times m$ matrix whose columns are the integer-valued ambiguities for each baseline, and $B$ is the $3 \times m$ matrix whose columns are the real-valued baseline coordinates. The noise on the matrix of observed-minus-computed observations is described making use of the vec operator, which stacks the columns of a matrix one under the other: the matrix $Q_{Y}$ describes the dispersion of the vector of observables $\operatorname{vec}(Y)$.

Aiming to estimate a platform's full attitude from the GNSS observations collected from three (or more) antennae mounted on one body, the model (12) is modified to include the attitude matrix as unknown. Assuming that the baseline coordinates $B$ in model (12) are derived in the $x y z$ orthogonal frame (usually the ECEF, Earth-Centered Earth-Fixed, or the ENU, East-North-Up frames are used), a rotation matrix $R$ is applied to convert $B$ into the local orthogonal frame uvw:

$$
R^{T} B=B_{u v w}
$$

The baseline coordinates in the local frame $B_{\text {uvw }}$ are assumed to be known and constant. The rotation matrix belongs to the class of orthogonal matrices $\mathbb{O}$; in order to maintain a full validity of the model when less than three baselines 
$(m<3)$ are available, the matrix $R$ is taken as (Teunissen, 2007a)

$$
\begin{aligned}
& \left\{\begin{array}{l}
m \geq 3 \\
q=3
\end{array} \quad: \quad R=\left[r_{1}, r_{2}, r_{3}\right]\right. \\
& \left\{\begin{array}{l}
m=2 \\
q=2
\end{array} \quad: \quad R=\left[r_{1}, r_{2}\right]\right. \\
& \left\{\begin{array}{l}
m=1 \\
q=1
\end{array} \quad: \quad R=\left[r_{1}\right]\right.
\end{aligned}
$$

where $r_{i}$ is a 3 -vector of unit length and $q$ is introduced for notational convenience. The orthonormality constraint on $R$ implies that $r_{i}^{T} r_{i}=1$, for $i=1,2,3$, and $r_{i}^{T} r_{j}=0$ for $i \neq j$, so that $R^{T} R=I_{q}$.

Introducing the rotation matrix as unknown in the model (12) gives the GNSSbased Attitude model (Teunissen, 2007a):

$$
\begin{aligned}
E(Y) & =A Z+G R B_{\text {uvw }} \quad Z \in \mathbb{Z}^{n \times m} ; R \in \mathbb{O}^{3 \times q} \\
D(\operatorname{vec}(Y)) & =Q_{Y}
\end{aligned}
$$

In this contribution the least-squares solution of model (15) is addressed and tested: the two unknowns are the integer-valued matrix of ambiguities $Z \in \mathbb{Z}^{n \times m}$ and the orthogonal attitude matrix $R \in \mathbb{O}^{3 \times q}$. The integral resolution of these unknowns from the set of GNSS code and phase observations allow the estimation of precise attitude angles, and the GNSS receiver(s) can estimate the attitude manoeuvres of the platform (see Figure 1) by updating the GNSS observables epoch by epoch. Although the method proposed can be directly extended to a multi-frequency, multi-constellation GNSS, this contribution focuses on the most challenging scenario when performing GNSS-based Attitude Determination: the single-epoch, single-frequency, unaided (i.e. GPSonly) scenario.

\section{The Integer Least-Squares solution}

Solving for the unknowns in model (15) has been firstly addressed in (Teunissen, 2007a), where the Least-Squares solution was given. The application of the Least-Squares principle to a set of linear(ized) equations where a subset of the unknowns is subject to an integer constraint was coined the Integer LeastSquares principle (ILS). ILS estimation is efficiently implemented through the LAMBDA method, which mechanizes the search for the ambiguities in the integer domain and provides ambiguities with the highest possible success rate (Teunissen, 1994a, 1997; Verhagen and Teunissen, 2006). 


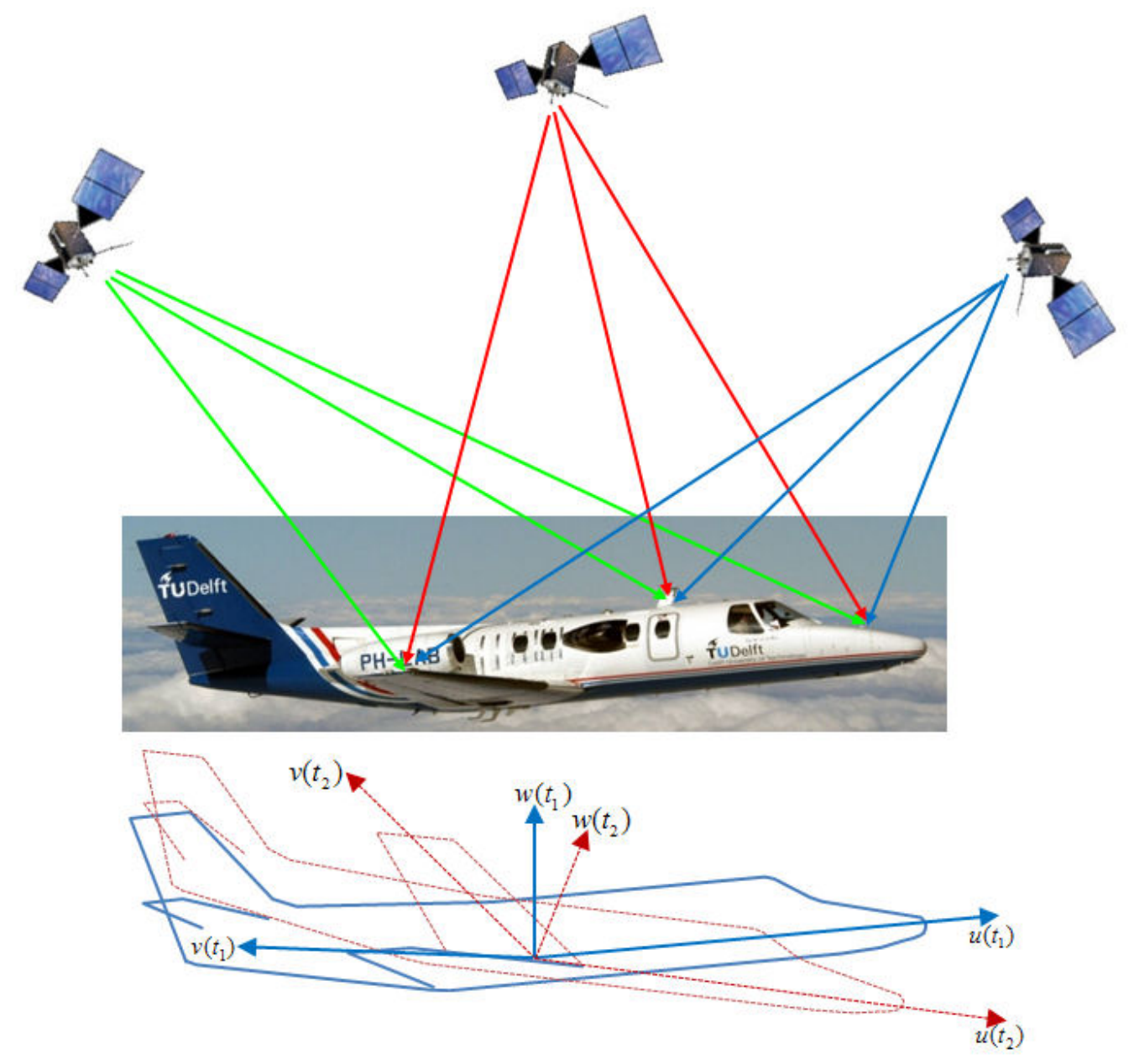

Fig. 1. The data collected from three or more GNSS antennae mounted on the aircraft fuselage and wings allow the estimation of the aircraft's attitude.

The extension of the ILS solution to problems subject to nonlinear geometrical constraints, such as the baseline length, was discussed in (Park and Teunissen, 2003; Teunissen, 2007b, 2008, 2009a), where the single-baseline case was examined. The solution of the Constrained ILS problem was given and implemented via an extension of the LAMBDA method, coined the Constrained LAMBDA (C-LAMBDA) method. The method was tested through simulations as well as through static and dynamic experiments (Buist, 2007; Giorgi et al., 2008; Giorgi and Buist, 2008; Park and Teunissen, 2008; Teunissen et al., 2009b). The solution given in this contribution is a multivariate generalization of these works: an arbitrary number of baselines can be included in the model and integrally solved for.

The application of the least-squares principle to (15) aims to minimize the weighted squared norm of the residuals while respecting the constraints posed on the unknowns:

$$
\min _{Z \in \mathbb{Z}^{n \times m} ; R \in \mathbb{O}^{3 \times q}}\left\|\operatorname{vec}\left(Y-A Z-G R B_{u v w}\right)\right\|_{Q_{Y}}^{2}
$$


The norm (16) is decomposed into a sum of squares as (Teunissen, 2007a)

$$
\begin{gathered}
\left\|\operatorname{vec}\left(Y-A Z-G R B_{u v w}\right)\right\|_{Q_{Y}}^{2}= \\
\left\|\operatorname{vec}(Y)-\left(I_{m} \otimes A\right) \operatorname{vec}(Z)-\left(B_{u v w}^{T} \otimes G\right) \operatorname{vec}(R)\right\|_{Q_{Y}}^{2}= \\
\|\operatorname{vec}(\hat{E})\|_{Q_{Y}}^{2}+\|\operatorname{vec}(Z-\hat{Z})\|_{Q_{\hat{Z}}}^{2}+\|\operatorname{vec}(\hat{R}(Z)-R)\|_{Q_{\hat{R}(Z)}}^{2}
\end{gathered}
$$

with $\|\cdot\|_{Q}^{2}=(\cdot)^{T} Q^{-1}(\cdot)$ and where $\otimes$ denotes the Kronecker product. The following property of the vec operator, $\operatorname{vec}\left(M_{1} M_{2} M_{3}\right)=\left(M_{3}^{T} \otimes M_{1}\right) \operatorname{vec}\left(M_{2}\right)$, has been used. $\hat{E}$ is the matrix of least-squares residuals.

The decomposition (17) makes use of the float solution, which is the leastsquares solution of (15) obtained by disregarding both the integer constraint on $Z$ and the orthonormality constraint on $R$ :

$$
\begin{aligned}
& N \cdot\left(\begin{array}{l}
\operatorname{vec}(\hat{Z}) \\
\operatorname{vec}(\hat{R})
\end{array}\right)=\left[\begin{array}{c}
I_{m} \otimes A^{T} \\
B_{u v w} \otimes G^{T}
\end{array}\right] Q_{Y}^{-1} \operatorname{vec}(Y) \\
& N=\left[\begin{array}{c}
I_{m} \otimes A^{T} \\
B_{u v w} \otimes G^{T}
\end{array}\right] Q_{Y}^{-1}\left[I_{m} \otimes A B_{u v w}^{T} \otimes G\right]
\end{aligned}
$$

Matrices $\hat{Z}$ and $\hat{R}$ are the float estimators of the integer ambiguity matrix $Z$ and the rotation matrix $R$, respectively. These float solutions do not generally respect the constraints: $\hat{Z}$ is real-valued and $\hat{R}$ is non-orthogonal. The v-c matrices of the float solutions are obtained by inverting the normal matrix,

$$
\left[\begin{array}{cc}
Q_{\hat{Z}} & Q_{\hat{Z} \hat{R}} \\
Q_{\hat{R} \hat{Z}} & Q_{\hat{R}}
\end{array}\right]=N^{-1}
$$

Would we assume the integer ambiguity matrix $Z$ as known, then the float estimator of the rotation matrix $R$ is obtained as

$$
\operatorname{vec}(\hat{R}(Z))=\operatorname{vec}(\hat{R})-Q_{\hat{R} \hat{Z}} Q_{\hat{Z}}^{-1} \operatorname{vec}(\hat{Z}-Z)
$$

Application of the variance propagation law to expression (20) gives the v-c matrix of $\hat{R}(Z)$ as:

$$
Q_{\hat{R}(Z)}=Q_{\hat{R}}-Q_{\hat{R} \hat{Z}} Q_{\hat{Z}}^{-1} Q_{\hat{Z} \hat{R}}
$$

It is the inverse of this matrix which is used as weight matrix in the last term of (17). The precision of $\hat{R}(Z)$ is considerably higher than that of $\hat{R}$, since it is now driven by the fixed carrier phase observations. Note that also the matrix $\hat{R}(Z)$ is generally not orthogonal. 
From expressions (16)-(17) it follows that the minimization problem that has to be solved is:

$$
\begin{gathered}
\check{Z}=\arg \min _{Z \in \mathbb{Z}^{n \times m}}\left[\|\operatorname{vec}(Z-\hat{Z})\|_{Q_{\hat{Z}}}^{2}+\|\operatorname{vec}(\hat{R}(Z)-\check{R})\|_{Q_{\hat{R}(Z)}}^{2}\right] \\
\check{R}=\arg \min _{R \in \mathbb{O}^{3 \times q}}\|\operatorname{vec}(\hat{R}(Z)-R)\|_{Q_{\hat{R}(Z)}}^{2}
\end{gathered}
$$

The integer minimizer $\check{Z}$ weighs the sum of two coupled terms: the first is the distance with respect to the float solution $\hat{Z}$ weighted by $Q_{\hat{Z}}^{-1}$, and the second is the distance between $\hat{R}(Z)$ and the solution of the nonlinear constrained leastsquares problem (23), weighted by $Q_{\hat{R}(Z)}^{-1}$. The final estimate of the platform's attitude is given by the rotation matrix $\check{R}$, which follows from minimizing in a weighted least-squares sense the distance of matrix $\hat{R}(Z)$ to an orthonormal matrix.

A closed-form solution for the minimizer (22) is not known, and a direct search in the space of integer matrices must be employed. The integer matrix $\check{Z}$ is searched inside the search space given by:

$$
\Omega\left(\chi^{2}\right)=\left\{Z \in \mathbb{Z}^{n \times m} \mid\|\operatorname{vec}(Z-\hat{Z})\|_{Q_{\hat{Z}}}^{2}+\|\operatorname{vec}(\hat{R}(Z)-\check{R})\|_{Q_{\hat{R}(Z)}}^{2} \leq \chi^{2}\right\}
$$

where $\chi$ is a scalar carefully chosen as to limit the set $\Omega\left(\chi^{2}\right)$ : its value should be large enough to guarantee the non-emptiness of the search space, but not too large to avoid an excessive computational load.

The set $\Omega\left(\chi^{2}\right)$ is searched in order to find the integer matrix $\check{Z}$ which returns the smallest value for the sum of the two terms in (22), and once it is found, the platform's attitude matrix $\check{R}$ is extracted. The process of integrally resolving for the integer ambiguity matrix $Z$ and the rotation matrix $R$ is the core of the proposed algorithm. The solution of (22) is based on an extension of the LAMBDA method, named the Multivariate Constrained LAMBDA (MCLAMBDA). The MC-LAMBDA method proceeds by minimizing a function which accounts for both the integer and the attitude matrix. This is different from how it is often done in practice, where the attitude is determined based on an estimation of the baseline vectors, by firstly solving for the ambiguities and then estimating the attitude matrix by solving (23). The constrained least square problem $(23)$, for $Q_{\hat{R}(Z)}$ diagonal, is the well known Wahba's problem (Wahba, 1965). The strengthening of the underlying GNSS model by including the additional orthonormality constraint enhances the capacity of correctly fixing the sought-for integer matrix, resulting in a much more reliable ambiguity resolution process. 
The MC-LAMBDA method uses the same principle as the original LAMBDA method to decorrelate the search space to allow a fast and efficient search, but it is modified to include the additional nonlinear geometrical constraints. Three steps are involved in the solution: first, the float estimates of the unknowns are derived as (18); then the search for the integer minimizer $\check{Z}$ is performed inside the (decorrelated) set $\Omega\left(\chi^{2}\right)$; finally the attitude matrix is extracted solving the nonlinear constrained problem in (22).

In the next sections the method is tested and its performance is presented. Particular attention is paid to assessing the capacity of fixing the correct integer ambiguities. The performance of the MC-LAMBDA method is compared with that of the standard unconstrained method, where the orthonormality constraint on $R$ in (22) is disregarded and the standard LAMBDA method is applied. If one disregards the constraint on the rotation matrix, it follows that the last term of (17) can be made zero for any choice of $Z$, and therefore the ambiguity resolution problem is decoupled from the one of attitude estimation. As a result, the minimization problem reduces to

$$
\begin{aligned}
& \check{Z}^{U}=\arg \min _{Z \in \mathbb{Z}^{n \times m}}\|\operatorname{vec}(Z-\hat{Z})\|_{Q_{\hat{Z}}}^{2} \\
& \check{R}^{U}=\arg \min _{R \in \mathbb{O}^{3 \times q}}\left\|\operatorname{vec}\left(\hat{R}\left(\check{Z}^{U}\right)-R\right)\right\|_{Q_{\hat{R}(Z)}}^{2}
\end{aligned}
$$

where firstly the ambiguities are resolved applying the standard (unconstrained) LAMBDA method and only then the attitude matrix is estimated solving the constrained least-squares problem.

\section{Testing the method}

The MC-LAMBDA method has been tested processing actual data collected during a static as well a dynamic experiment. On 1 November 2007 a flight test was performed, as part of the Gravimetry using Airborne Inertial Navigation (GAIN) project (Alberts et al., 2008). Several GNSS receivers were employed both on the ground, to set up a ground station to provide a Real Time Kinematic (RTK) solution for the aircraft's position (Buist, 2008), and onboard the aircraft, to estimate its attitude. The experiment aimed to investigate the local gravity acceleration variations over an area spanning several tens of kilometers: to this purpose the aircraft was equipped with an Inertial Navigation System (INS), which output is used to test the GNSS-based Attitude estimation accuracy in this contribution.

The next two sections review the set-up of the ground station and aircraft, and the testing results are given. Two performance parameters have been investigated: the unaided, single-epoch, single-frequency success rate, i.e. the ratio 
of correctly fixed ambiguities based on a single-epoch of observations tracking GNSS satellites on a single frequency (and consequently the availability of a precise GNSS-based attitude solution on a single-epoch base), and the accuracy of the attitude angles.

All the angles derived are referred to the ENU (East-North-Up) frame (see Figure 2), with the Heading angle $\psi \in\left[-180^{\circ} ;+180^{\circ}\right]$, relative to the North direction. The rotation matrix is parameterized in term of the three Euler angles Heading $(\psi)$, Elevation $(\theta)$ and Bank $(\phi)$, and it is obtained as a succession of three rotations around the main axis: $R(\psi, \theta, \phi)=R_{3}(\psi) R_{2}(\theta) R_{1}(\phi)$. The local frame $B_{u v w}$ is chosen as to have the first axis $u$ aligned with the first baseline, the second $v$ perpendicular to $u$, in the plane formed by the first two baselines, and the third axis $w$ perpendicular to $u$ and $v$, directed as to form a right-handed orthogonal frame.

\subsection{A static test: processing the ground station data}

A set of three geodetic quality receivers (a Trimble R7 and two Trimble SSi) and three antennae (a Trimble Zephyr Geodetic L1/L2, the Master, and two Trimble Geodetic W Groundplane, the auxiliaries) were used to set up a ground station. The Trimble R7 was connected to the Trimble Zephyr Geodetic, which was placed above a known static reference point; the other two antennae were placed in proximity of the first one at a known fixed distance (see Figure 3). Data were collected between 10:44 and 13:29, UTC time, at the frequency of $1 \mathrm{~Hz}$, so that a total of 9915 epochs were logged.

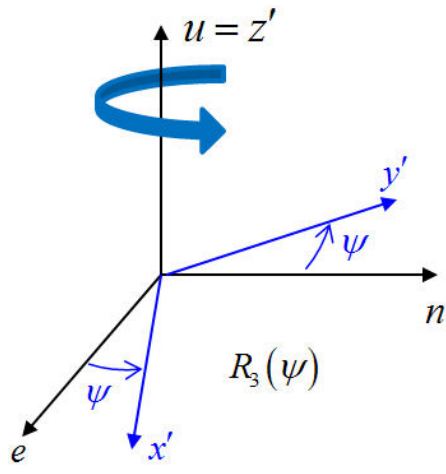

Heading

enu $\Rightarrow x^{\prime} y^{\prime} z^{\prime}$

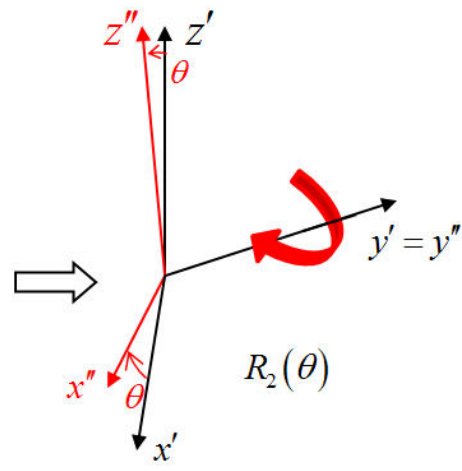

Elevation

$x^{\prime} y^{\prime} z^{\prime} \Rightarrow x^{\prime \prime} y^{\prime \prime} z^{\prime \prime}$

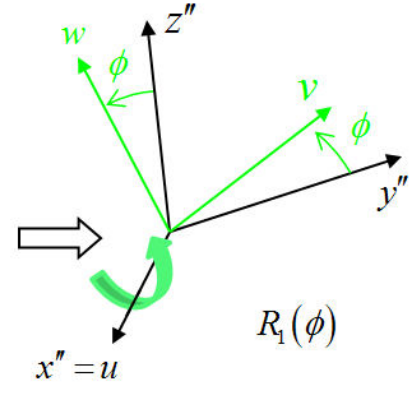

Bank

$x^{\prime \prime} y^{\prime \prime} z^{\prime \prime} \Rightarrow u v w$

Fig. 2. The three consecutive rotations which transform the coordinates from the ENU frame (enu) to the local (body) frame uvw. 


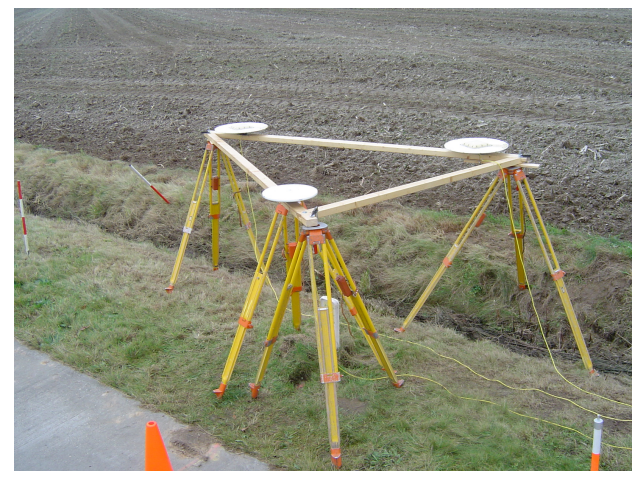

(a) Picture of the ground station setup.

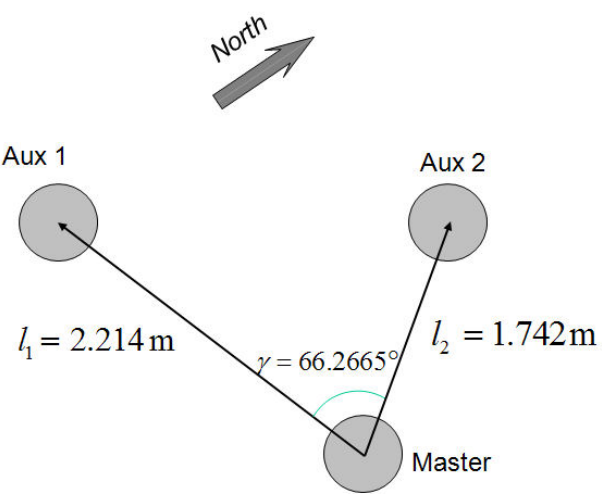

(b) Scheme of the Ground Station set-up, reporting the two baselines lengths and the angle between them, as surveyed on field.

Fig. 3. The Ground Station set-up.

Table 1 reports the single-frequency, single-epoch success rate obtained processing the static dataset with both the LAMBDA and the MC-LAMBDA methods, as function of the number of satellites tracked. The MC-LAMBDA method shows a large robustness, obtaining a successful fixing (success rate higher than 99\%) in all but one condition, and providing the correct precise attitude solution for all the epochs processed when five or more satellites are tracked. When only four satellites are tracked, the MC-LAMBDA algorithm still provides a success rate higher than $80 \%$ : the lower performance is mainly due to the bad geometry of the four satellites tracked, for which the PDOP value is higher than 17 . The number of available satellites strongly affects the performance of the standard LAMBDA method, whereas the inclusion of the geometrical constraints strengthens the model such to guarantee a large fixing rate in harsher conditions.

Table 1

The single-frequency, single-epoch success rate for the LAMBDA and the MCLAMBDA methods (\%) as function of the number of satellites tracked

\begin{tabular}{ccc} 
Number of tracked satellites (PDOP) & LAMBDA [\%] & MC-LAMBDA [\%] \\
\hline $9(2.0)$ & 99.84 & 100 \\
$8(2.1)$ & 97.73 & 100 \\
$7(2.2)$ & 78.47 & 100 \\
$6(2.6)$ & 38.91 & 100 \\
$5(3.2)$ & 4.98 & 99.57 \\
$4(17.1)$ & 0.88 & 84.22 \\
\hline
\end{tabular}


Table 2

Standard deviation of the three estimated attitude angles as function of the baseline coordinate frame chosen.

\begin{tabular}{cccc} 
Frame & $\sigma(\psi)[\mathrm{deg}]$ & $\sigma(\theta)[\mathrm{deg}]$ & $\sigma(\phi)[\mathrm{deg}]$ \\
\hline$B_{u v w}^{\prime}$ & 0.054 & 0.120 & 0.174 \\
$B_{u v w}^{\prime \prime}$ & 0.053 & 0.186 & 0.100 \\
\hline \hline
\end{tabular}

The two baselines Main-Aux $x_{1}$ and Main-Aux 2 determine the local baseline frame $B_{u v w}$ : the precision of the estimated attitude angles depends on how the frame $B_{u v w}$ is chosen, since longer baselines provide more precise estimations. Table 2 shows the precision of the estimated attitude angles with different

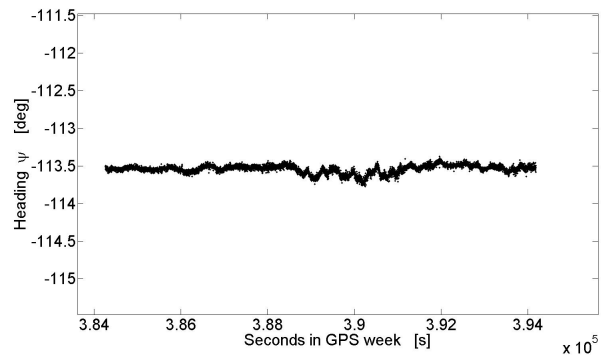

(a) Heading $\psi(t), B_{u v w}^{\prime}$

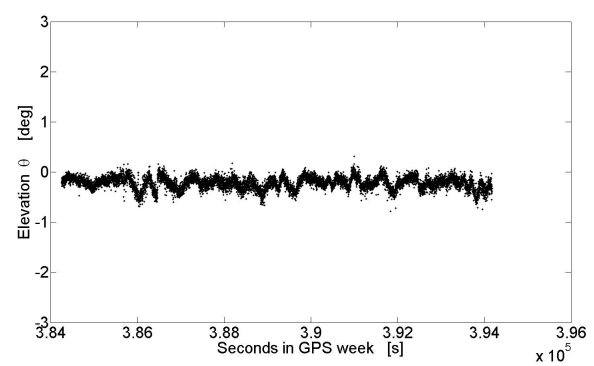

(c) Elevation $\theta(t), B_{u v w}^{\prime}$

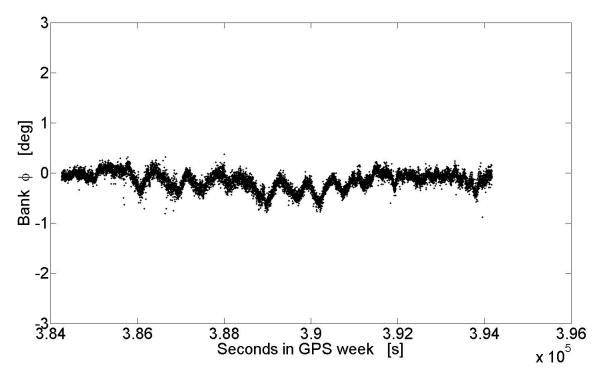

(e) Bank $\phi(t), B_{u v w}^{\prime}$

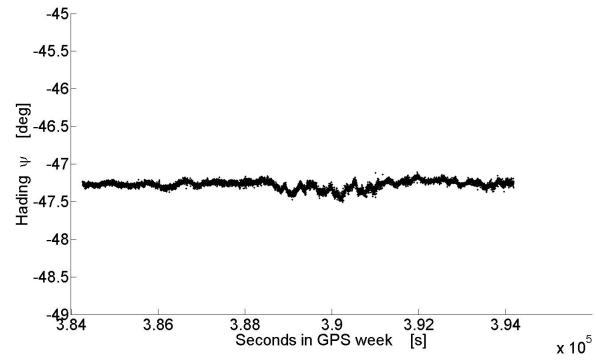

(b) Heading $\psi(t), B_{u v w}^{\prime \prime}$

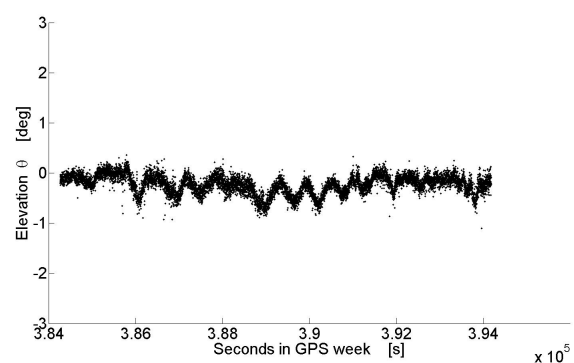

(d) Elevation $\theta(t), B_{u v w}^{\prime \prime}$

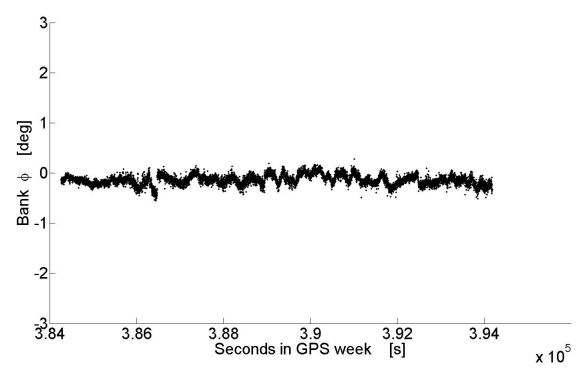

(f) Bank $\phi(t), B_{u v w}^{\prime \prime}$

Fig. 4. The time series of the estimated static attitude angles. $B_{u v w}^{\prime}$ is the local baseline coordinates frame built as to have the longest baseline aligned with the axis $u$, while $B_{u v w}^{\prime \prime}$ is the local baseline coordinates frame built as to have the shortest baseline aligned with the axis $u$. 
choices for the baseline coordinate frame:

$$
B_{u v w}^{\prime}=\left[\begin{array}{cc}
2.214 & 0.701 \\
0 & 1.595
\end{array}\right][m] \quad B_{u v w}^{\prime \prime}=\left[\begin{array}{cc}
1.7422 & 0.891 \\
0 & 2.026
\end{array}\right][m]
$$

where $B_{u v w}^{\prime}$ is chosen as to have the longer baseline Main-Aux 1 aligned with the first axis, while the $u$ axis of the frame $B_{u v w}^{\prime \prime}$ is aligned with the shorter baseline Main - Aux $x_{2}$. Both cases show a higher precision of the estimated heading angles, which are less affected by the GNSS satellite geometry (the satellites are observed only from one side of the sky, causing a larger propagation of the errors in the vertical direction rather than in the local horizontal plane). The elevation and bank angles are estimated with lower precision, and the dependence on the baseline length is clear: for $B_{u v w}^{\prime}$, the second baseline is shorter, causing lower precision in the bank estimation, while for $B_{u v w}^{\prime \prime}$, with the second baseline being longer, the bank angle estimation is more precise. Figure 4 shows the time series of the estimated attitude angles for the two choices of local baseline coordinates.

\subsection{A dynamic test: aircraft attitude estimation}

As a support for the GAIN project, the Cessna Citation II of the Faculty of Aerospace Engineering, Delft University of Technology, was equipped with a number of GNSS antennae: two on the body, approximately in the middle of the fuselage (a Novatel AIL DM-C L1-L2 and a L1/L2 Sensor Systems), one at the extremity of the left wing, and one on the nose (both L1 Sensor Systems). One of the antennae on the fuselage and the two on the nose and

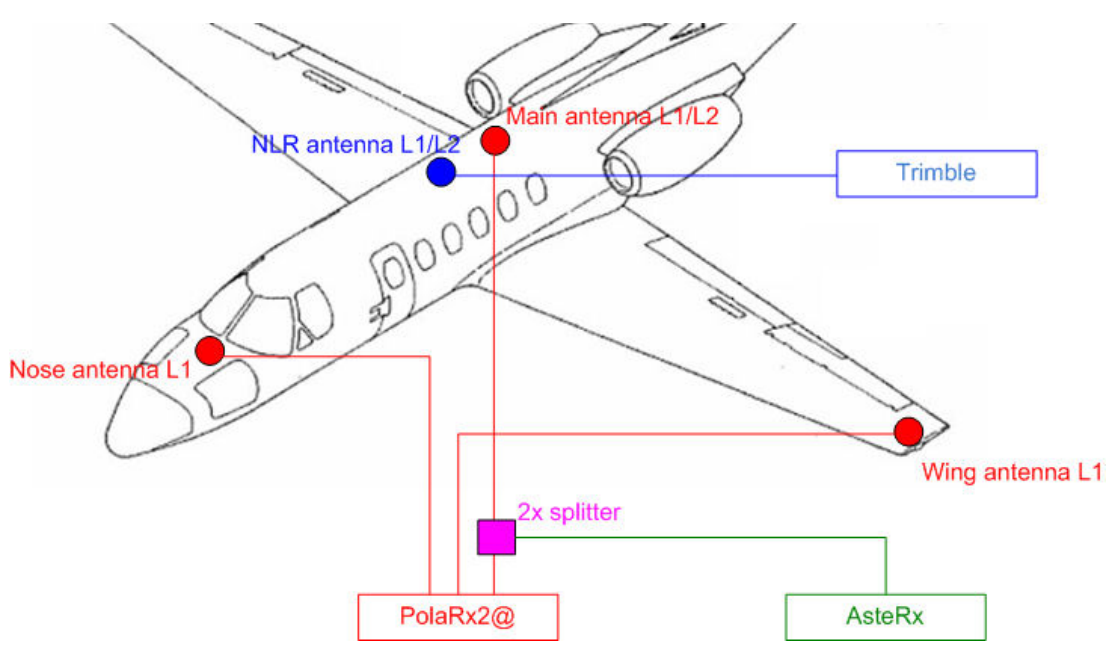

Fig. 5. The set up of the GNSS antennae and receivers on the Cessna Citation II. 


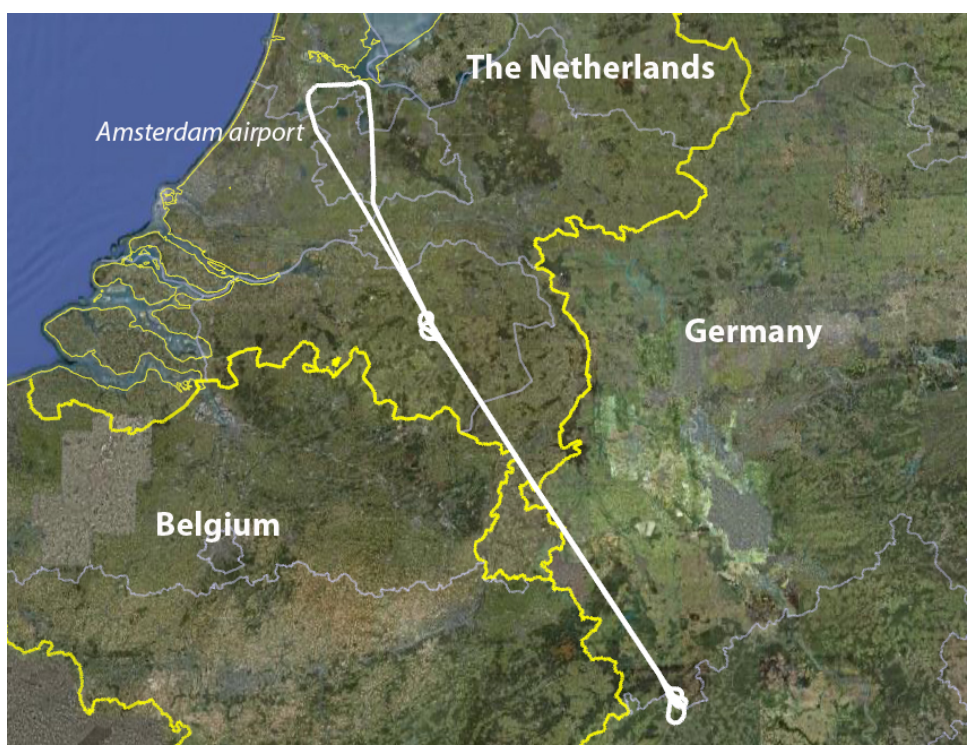

(a) The ground track of the flight.

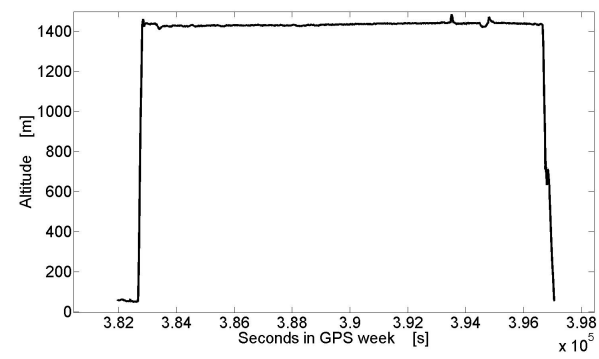

(b) The altitude profile of the flight.

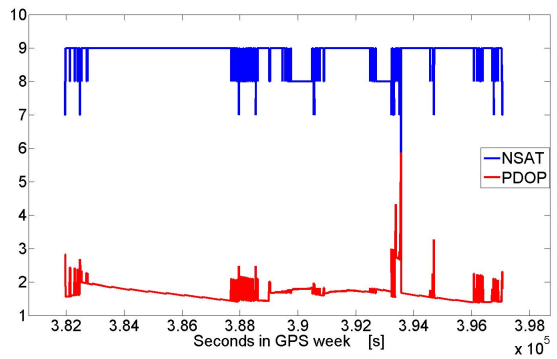

(c) The number of tracked satellites and PDOP values during the aircraft test.

Fig. 6. The ground track and altitude profile of the flight, and the number of tracked satellites and PDOP.

the wing were connected to a Septentrio PolaRx2@ receiver, logging data for the entire duration of the flight, from 10:06 to 14:18 (UTC time), collecting a total of 15101 epochs (at $1 \mathrm{~Hz}$ ). Figure 5 shows the set up of the antennae and receivers on the Cessna Citation II: only the data logged from the Septentrio receiver are used in this analysis. The body frame is built so to have the first axis $u$ aligned with the baseline formed by the antennae on the body and the nose:

$$
B_{\text {uvw }}=\left[\begin{array}{cc}
4.90 & -0.39 \\
0 & 7.60
\end{array}\right] \quad[\mathrm{m}]
$$

The sensing equipment carried onboard for the gravimetry study was an Inertial Navigation System (INS): the Honeywell Laseref II IRS (YG1782B). Figure 6 shows the ground track of the flight calculated with the singlefrequency observations collected on the main antenna; also the altitude profile, the number of satellites tracked and the corresponding PDOP values are shown. 
Table 3

The single-frequency, single-epoch success rate for the LAMBDA and the MCLAMBDA methods (\%) and the standard deviations of the differences between GPS and INS attitude angles output.

LAMBDA [\%] MC-LAMBDA [\%]

Single-epoch, single-frequency success rate 31.68 88.10

$\begin{array}{ll}\sigma(\psi)[\mathrm{deg}] & 0.065 \\ \sigma(\theta)[\mathrm{deg}] & 0.202 \\ \sigma(\phi)[\mathrm{deg}] & 0.124\end{array}$

The data have been processed on a single-epoch base, and no external aid, validation or quality control procedure have been applied. The unaided, singleepoch, single-frequency success rate for the entire duration of the flight is reported in table 3 . The improvement when employing the Multivariate Constrained LAMBDA method is very impressive: the estimation of the integer ambiguities is successful for more than $88 \%$ of the time, thus making available a reliable attitude estimation almost epoch-by-epoch. The importance of the obtained result is evident when considering that a fast recovery after a cycle-slip or carrier loss-of-lock is of utmost importance for those applications that require a continuous knowledge of the platform's attitude. Reducing the number of epochs needed to guarantee a reliable solution, ideally to a single epoch, is then a primary requirement.

Figure 7 shows the time series of the three attitude angles; the INS output is also reported, to provide a term of comparison. The accuracy of the solution can be approximatively determined by comparing the attitude angles provided by the given algorithm and the output of the INS: table 3 reports the standard deviations of the differences between the angles provided by the GPS and the INS. Similar to the static experiment, the heading angle can be determined with higher precision: the differences with respect to the INS output are less than 0.07 degrees $(1 \sigma)$. The elevation angle is the least precise, presenting a noisier characteristic; the bank angle, thanks to the longer baseline length, could be determined more precisely than the elevation, with differences respect to the INS output contained within 0.13 degrees $(1 \sigma)$.

\section{Conclusion}

GNSS is an important technology for providing accurate position and attitude estimations of Remote Sensing platforms. This contribution focussed on GNSS carrier phase-based Attitude Determination, where we analyzed the 


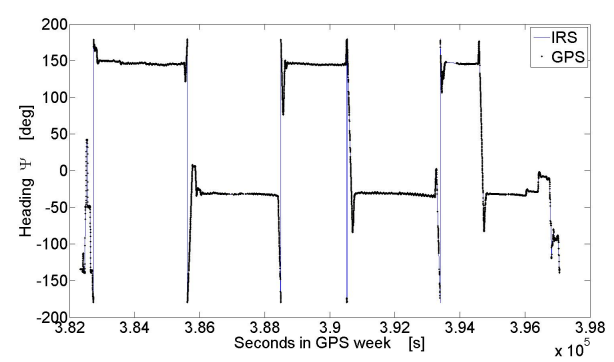

(a) Heading $\psi(t)$

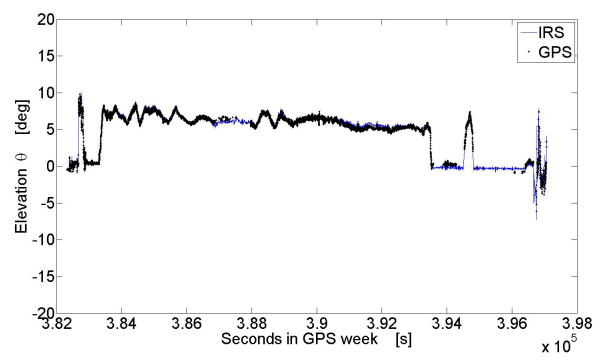

(c) Elevation $\theta(t)$

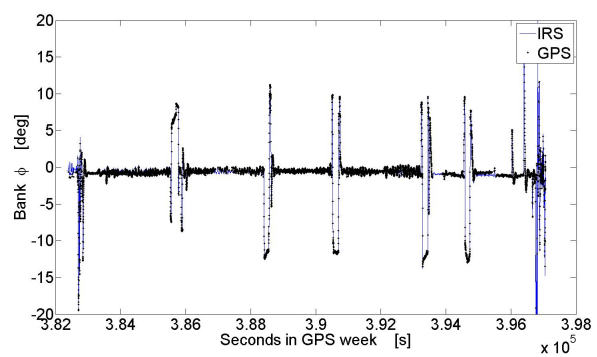

(e) Bank $\phi(t)$

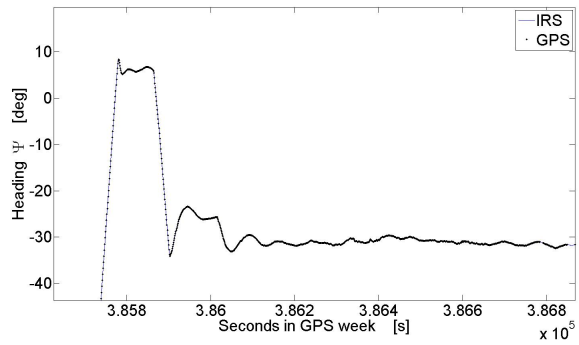

(b) Heading $\psi(t)$, zoom

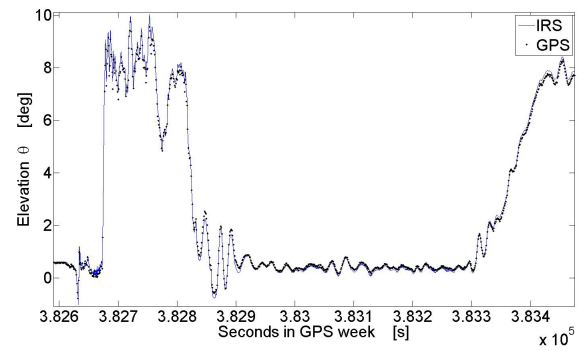

(d) Elevation $\theta(t)$, zoom

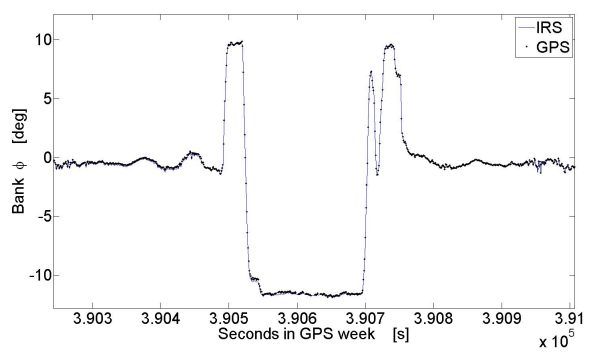

(f) Bank $\phi(t)$, zoom

Fig. 7. Time series of the three attitude angles as estimated via GNSS and provided by the INS. The whole processed time span is shown on the left, while on the right a shorter interval is visualized.

performance of a novel method for integral ambiguity resolution and attitude estimation of flying platforms.

Integer ambiguity resolution is the key for being able to exploit the very high precision of the carrier phase data for attitude determination. In this contribution we described the GNSS attitude model and presented the corresponding multivariate constrained integer least-squares solution. Our method for computing this solution is a multivariate constrained version of the LAMBDA method. The method presented is generally applicable and therefore not restricted to a particular GNSS application. It is characterized by the fact that it is independent of baseline length, independent of platform dynamics, and independent of which GNSS is chosen, therefore applicable to any geometrical arrangement of antennae, collecting data from any single or multiple GNSS. The principles of the new Multivariate LAMBDA method are illustrated and its performance tested using data collected during an airborne remote sensing campaign, focusing on the most challenging scenario: single-frequency, single- 
epoch, unaided (i.e. GPS-only) full attitude ambiguity resolution. Tests were performed processing both data collected on a static platform, with high quality receivers and antennae, and data collected on a dynamic platform, affected by higher noise levels and multipath. The superior success rate performance compared to the ones of the unconstrained standard LAMBDA method are due to the rigorous incorporation of the nonlinear constraints into the integer estimation process. These constraints are given by the known body frame geometry of the GNSS antennae configuration. The strengthening of the model leads to a very robust method, capable of providing precise attitude estimation in a wider range of conditions (lower number of satellites, higher noise, multipath-affected observations). The given method is suitable for marine, airborne as well as spaceborne remote sensing campaigns, where a reliable method to resolve the GNSS integer ambiguities is required.

\section{Acknowledgment}

The second author P.J.G. Teunissen is the recipient of an Australian Research Council Federation Fellowship (project number FF0883188): this support is gratefully acknowledged.

The research of S. Verhagen is supported by the Dutch Technology Foundation STW, applied science division of NWO and the Technology Program of the Ministry of Economic Affairs.

The GAIN experiment team, a mutual cooperation between chairs of Control and Simulation, Physical and Space Geodesy and Mathematical Geodesy and Positioning at Delft University of Technology is acknowledged for the pleasant cooperation during the flight described in this paper.

\section{References}

Alberts, B. A., B. C. Gunter, A. Muis, Q. P. Chu, G. Giorgi, L. Huisman, P. J. Buist, C. C. J. M. Tiberius, and H. Lindenburg (2008). Correcting Strapdown GPS/INS Gravimetry Estimates with GPS Attitude Data. Presented at the International Symposium on Gravity, Geoid and Earth Observation, GGEO 2008, Chania, Crete, Greece, 23-27 June 2008.

Axelrad, P. and L. Ward (1994). On-Orbit GPS Based Attitude and Antenna Baseline Estimation. Proceedings of ION-NTM, San Diego, CA, US, 441450.

Azpilicueta, F., C. Brunini, and S. M. Radicella (2006). Global Ionospheric Maps from GPS Observations Using Modip Latitude. Advances in Space Research 38(11), 2324-2331.

Bar-Itzhack, I. Y., P. Montgomery, and J. Garrick (1998). Algorithm for Atti- 
tude Determination using Global Positioning System. Journal of Guidance, Control and Dynamics 21(6), 846-852.

Beutler, G., M. Rothacher, S. Schaer, T. A. Springer, J. Kouba, and R. E. Neilan (1999). The International GPS Service (IGS): an Interdisciplinary Service in Support of earth Sciences. Advances in Space Research 23(4), 631-653.

Bock, H., U. Hugentobler, T. A. Springer, and G. Beutler (2002). Efficient Precise Orbit Determination of LEO Satellites Using GPS. Advances in Space Research 30(2), 295-300.

Bock, H., A. Jaggi, D. Svehla, G. Beutler, U. Hugentobler, and P. Visser (2007). Precise Orbit Determination for the GOCE Satellite Using GPS. Advances in Space Research 39(10), 1638-1647.

Brown, R. A. (1992). Instantaneous GPS Attitude Determination. Proceedings of IEEE Position Location and Navigation Symposium, PLANS 92, US, $113-120$.

Buist, P. J. (2007). The Baseline Constrained LAMBDA Method for Single Epoch, Single Frequency Attitude Determination Applications. Proceedings of ION GPS, Fort Worth, TX, US, 2962-2973.

Buist, P. J. (2008). GNSS Kinematic Relative Positioning for Spacecraft: Data Analysis of a Dynamic Testbed. Proceedings of 26th ISTS (International Symposium on Space Technology and Science, Hamamatsu, Japan.

Cohen, C. E. (1992). Attitude Determination Using GPS. Ph.D. Thesis, Stanford University, Palo Alto, CA, USA.

Corbett, S. (1993). GPS for Attitude Determination and Positioning in Airborne Remote Sensing. Proceedings of ION GPS, Salt Lake City, UT, US, 789-796.

Crassidis, J. L., F. L. Markley, and E. G. Lightsey (1997). A New Algorithm for Attitude Determination Using Global Positioning System Signals. AIAA Journal of Guidance, Control, and Dynamics 20(5), 891-896.

Dai, L., K. V. Ling, and N. Nagarajan (2004). Real-time Attitude Determination for Microsatellite by LAMBDA Method Combined with Kalman Filtering. Proceedings of 22nd AIAA International Communications Satellite Systems Conference and Exhibit 2004 (ICSSC), Monterey, California, US.

Euler, H. J. (1995). Attitude determination: Exploiting All Information for Optimal Ambiguity Resolution. Proceedings of ION GPS, 1751-1757.

Giorgi, G. and P. J. Buist (2008). Single-epoch, Single Frequency, Standalone Full Attitude Determination: Experimental Results. Presented at 4th ESA Workshop on Satellite Navigation User Equipment Technologies, NAVITEC08, ESA-ESTEC, The Netherlands.

Giorgi, G., P. J. G. Teunissen, and P. J. Buist (2008). A Search and Shrink Approach for the Baseline Constrained LAMBDA: Experimental Results. Proceedings of the International Symposium on GPS/GNSS 2008. A. Yasuda (Ed.), Tokyo University of Marine Science and Technology, 797-806.

Hauschild, A. and O. Montenbruck (2007). GPS-Based Attitude Determina- 
tion for Microsatellite. Proceedings of ION GNSS, Forth Worth, TX, USA, 2424-2434.

Jin, S.G., J. Park, J. Cho, and P. Park (2007). Seasonal Variability of GPSderived Zenith Tropospheric Delay (1994-2006) and Climate Implications. Journal of Geophysical Research 112(D09110).

Jin, S.G. and O.F. Luo (2009). Variability and Climatology of PWV from Global 13-year GPS Observations. IEEE Transactions on Geoscience and Remote Sensing 47(7), 1918-1924.

Kang, Z., P. Nagell, and R. Pastor (2003). Precise Orbit Determination for GRACE. Advances in Space Research 31(8), 1875-1881.

Kim, D. and R. B. Langley (2000). GPS Ambiguity Resolution and Validation: Methodologies, Trends and Issues. Presented at the 7th GNSS Workshop - International Symposium on GPS/GNSS, Seoul, Korea, Nov. 30-Dec. 2, 2000.

Knedlik, S., E. Edwan, J. Zhou, Z. Dai, P. Ubolkosold, and O. Loffeld (2008). GPS/INS Integration for Footprint Chasing in Bistatic SAR Experiments. IGARSS 2008, Geoscience and Remote Sensing Symposium 3(3), 459-462.

Kocaman, S. (2003). GPS and INS Integration with Kalman Filtering for Direct Georeferencing of Airborne Imagery. Geodetic Seminar Report, Institute of Geodesy and Photogrammetry, ETH Honggerberg, Zurich.

Kuylen, L. V., F. Boon, and A. Simsky (2005). Attitude Determination Methods Used in the PolarRx2@ Multi-antenna GPS Receiver. Proceedings of ION GPS, Long Beach, CA, US, 125-135.

Kuylen, L. V., P. Nemry, F. Boon, A. Simsky, and J. F. M. Lorga (2006). Comparison of Attitude Performance for Multi-Antenna Receivers. European Journal of Navigation 4 (2), 1-9.

Legat, K., J. Skaloud, and P. Schaer (2006). Real-Time Processing of GPS/INS Data for On-the-fly Quality Control in Airborne Mobile Mapping. Presented at the GNSS European Navigation Conference, ENC-GNSS 2006, Manchester, May \%-10.

Li, Y., K. Zhang, C. Roberts, and M. Murata (2004). On-the-fly GPS-based Attitude Determination Using Single- and Double-Differenced Carrier Phase Measurements. GPS Solutions 8, 93-102.

Madsen, J. and E. G. Lightsey (2004). Robust Spacecraft Attitude Determination Using Global Positioning System Receivers. Journal of Spacecraft and Rockets 41(4), 635-643.

Monikes, R., J. Wendel, and G. F. Trommer (2005). A Modified LAMBDA Method for Ambiguity Resolution in the Presence of Position Domain Constraints. Proceedings of ION GPS, Long Beach, CA, US, 18-24.

Montenbruck, O., M. Markgraf, M. Garcia-Fernandez, and A. Helm (2008). GPS for Microsatellites Status and Perspectives. Small Satellites for Earth Observation, R.Sandau et al. (eds.), Springer Netherlands.

Park, C. and P. J. G. Teunissen (2003). A New Carrier Phase Ambiguity Estimation for GNSS Attitude Determination Systems. Proceedings of International GPS/GNSS Symposium, Tokyo. 
Park, C. and P. J. G. Teunissen (2008). A Baseline Constrained LAMBDA Method for Integer Ambiguity Resolution of GNSS Attitude Determination Systems. Journal of Control, Robotics and Systems (in Korean) 14(6), 587594.

Psiaki, M. L. (2006). Batch Algorithm for Global-Positioning-System Attitude Determination and Integer Ambiguity Resolution. Journal of Guidance, Control, and Dynamics 29(5), 1071-1079.

Ruzhin, Y. Y., I. I. Shagimuratov, V. E. Kunitsyn, A. K. Depueva, and O. G. Razinkov (1998). GPS-Based Tomographic Reconstruction of the Ionosphere. Advances in Space Research 21(3), 521-524.

Schleppe, J. (1997). A Real-Time Attitude System Using a Quaternion Parameterization. Proceedings of KIS97, 395-408.

Teunissen, P. J. G. (1994a). A New Method for Fast Carrier Phase Ambiguity Estimation. Proceedings of IEEE Position Location and Navigation Symposium, PLANS '94, US, 562-573.

Teunissen, P. J. G. (1994b). Integer Least-Squares Estimation of the GPS Phase Ambiguities. Proceedings of the International Symposium on Kinematic Systems in Geodesy, Geomatics and Navigation, Banff, Canada, 221231.

Teunissen, P. J. G. (1997). A Canonical Theory for Short GPS Baselines. Part IV: Precision versus Reliability. Journal of Geodesy 71 (9), 513-525.

Teunissen, P. J. G. (1999). An Optimality Property of the Integer LeastSquares Estimator. Journal of Geodesy 73(11), 587-593.

Teunissen, P. J. G. (2007a). A General Multivariate Formulation of the MultiAntenna GNSS Attitude Determination Problem. Artificial Satellites 42(2), 97-111.

Teunissen, P. J. G. (2007b). The LAMBDA Method for the GNSS Compass. Artificial Satellites 41(3), 89-103.

Teunissen, P. J. G. (2008). GNSS Ambiguity Resolution for Attitude Determination: Theory and Method. Proceedings of the International Symposium on GPS/GNSS 2008. A. Yasuda (Ed.), Tokyo University of Marine Science and Technology.

Teunissen, P. J. G. (2009a). Integer Least Squares Theory for the GNSS Compass. Journal of Geodesy, Springer, submitted.

Teunissen, P. J. G., G. Giorgi, and P. J. Buist (2009b). Testing of a New Single-Frequency GNSS Carrier-Phase Compass Method: Land, Ship and Aircraft Experiments. GPS Solutions, Springer, submitted.

Teunissen, P. J. G. and A. Kleusberg (1998). GPS for Geodesy. Springer, Berlin Heidelberg New York.

Verhagen, S. and P. J. G. Teunissen (2006). New Global Navigation Satellite System Ambiguity Resolution Method Compared to Existing Approaches. Journal of Guidance, Control, and Dynamics 29(4), 981-991.

Wahba, G. (1965). Problem 65-1: A Least Squares Estimate of Spacecraft Attitude. SIAM Review 7(3), 384-386.

Wang, B., L. Miao, S. Wang, and J. Shen (2009). A Constrained LAMBDA 
Method for GPS Attitude Determination. GPS Solutions 13, 97-107. 